\title{
Acquired hearing loss
}

\author{
Dobriyanova V. \\ Throat Diseases Department, Faculty of Medicine at Sofia Medical University
}

SNHL - Sensorineural Hearing Loss

BAEP - Brainstem Auditory Evoked Potentials

I dentifying the causes for deafness can provide prognostic information, help in developing a rehabilitation plan and contribute to treatment or a patient's prognosis. Depending on the onset of hearing loss as concerning speech development it can be classified as prelingual, occurring before speech is developed and usually involving severe deafness (hearing thresholds from 61 to $90 \mathrm{~dB}$ for speech frequencies) and profound deafness (tone thresholds above 91dB), and postlingual - occurring gradually after normal speech has developed. In cases of postlingual deafness communication ability is not necessarily affected after loss of hearing.

Acquired hearing loss is a complex problem due to various reasons. The majority of cases are caused by external factors such as infections, diseases and trauma or can be age-related.

Infectious causes leading to deafness can occur before and after birth.

Neurotropic viruses such as herpes simplex, cytomegalovirus and rubella are well-known aetiological reasons for congenital sensorineural hearing loss and presumed causes for acquired hearing loss in acute viral infection. Mechanisms of action include direct viral invasion of the cochlea or the cochlear nerve, reactivation of a latent virus in the spiral ganglion and immune-mediated mechanisms such as mimicry, after an infection becomes systemic [1].

HSV-1 and HSV-2 can also cause both congenital and acquired SNHL. These are encapsulated, double-stranded DNA viruses from the Herpes virus family. The infection results from contact between secretions containing the virus and mucous membranes or damaged skin. The initial infection can be asymptomatic. HSV-1 infections are associated with a post-infection hearing loss or reactivation of the infection in early childhood. SNHL caused by $\mathrm{HSV}-1$ infection is more common than an HSV-2 one. If a herpes simplex infection is confirmed in young children, an audiometry is recommended.

Audiological and vestibular symptoms can also occur in people infected with Human immunodeficiency virus (HIV). It includes conductive hearing loss (often due to acute otitis media), sensorineural hearing loss, tinnitus, vertigo, ataxia [2, 3, 4]. Deafness can appear as the result of direct infection of the inner ear with HIV or indirectly after developing an opportunistic infection (For example: with CMV, syphilis, tuberculosis, cryptococcal meningitis) or ototoxic drug therapy.

HIV can also affect the central and peripheral part of the auditory system and the virus is detected in auditory and vestibular sensory cells, in strial cells and in the tectorial membrane [5]. Patients with HIV infection have abnormal BAEP, which implies engagement of the auditory nerve [6].

Epidemic parotitis caused by a virus belonging to Paramyxoviridae family, is characterised by unilateral or bilateral affecting of the parotid glands and complications, one of which can be sensorineural hearing loss. According to a study performed in Japan in 2009, cases of deafness after epidemic parotitis indicates $0.1 \%$ cases with unilateral hearing loss, severe hearing loss to deafness. The Centers for Control and Prevention of Diseases in the USA state that incidents are below 1\% [7]. Hearing loss associated with epidemic parotitis can appear before or after the infection in asymp- 
tomatic patients as well. Deafness associated with measles, mumps and rubella has decreased after the widespread use of the live attenuated vaccine.

The measles virus belongs to the family of the Paramyxovirus family. Hearing loss is a frequent complication of the measles infection. Before the widespread use of vaccination, measles accounted for 5 to $10 \%$ of the cases of deafness in the United States [8]. At present, it continues to be a common cause for profound hearing loss in areas where vaccination is rare [9].

Hearing loss resulting from measles is typically bilateral, moderate to severe and can follow measles encephalitis [8]. Measles is related to a high rate of otitis media (in $8.5-25 \%$ of the infected people), due to a temporary decrease in immunity. An after-effect of bacterial superinfection might lead to some cases of hearing loss associated with the infection.

Varicella Zoster Virus, VZV, is part of the Herpesviridae family. Initially, VZV caused a primary infection that can have expressed symptoms or be asymptomatic. Afterwards the virus can remain latent for a long period, in the neurons in different parts of the body. Reactivation of the latent VZV in ganglion geniculi causes Ramsay Hunt syndrome or herpes zoster oticus through the development of inflammation of the ganglion and inflammation of the facial nerve. Involvement of cranial nerve VIII ensues from transfer of the virus from the ganglion which is nearly located or directly from the facial nerve in the internal auditory canal. Symptoms include paralysis of the facial nerve, herpetiform vesicles, severe otalgia, SNHL (24\% of the affected patients), tinnitus and vertigo [10].

SNHL is unilateral and can vary from mild, high frequency to profound hearing loss but is usually mild to moderate. It can sometimes occur suddenly as a first manifestation of herpes zoster oticus. Patients with HZO have changes in BAEP followed by cochlear and retrocochlear dysfunction [11].

Haemorrhage of the cochlear nerve and destruction of the apex of the organ of Corti can be observed. Loss of cells of the ganglion geniculi with neuronal swelling and chromatolysis, perivascular lymphocytic infiltrate, nerve demyelianation and axonal loss are also observed. Hearing loss following HZO can be reversed after treatment while recovery from facial paralysis is reported less frequently. Severity of the facial paralysis not always correlates with the severity and prognosis of the auditory and vestibular symptoms.

Permanent hearing impairment occurs in about 5\% of the cases of HZO [12]. In these cases SNHL is caused by damage in the cochlea and in the more proximal auditory pathways. Hearing aids can be used to improve mild to moderate deafness (socially appropriate hearing to profound hearing loss). Other viral infections such as West Nile Virus, the human parvovirus B19, lymphocitic choriomeningitis virus are potentially connected with acquired hearing loss including that with sudden hearing loss.

\section{Meningitis}

Hearing loss is a well-documented complication of bacterial meningitis. Systematic review and meta-analysis of 132 articles has reported $33.6 \%$ frequency of hearing loss, due to bacterial meningitis caused by various agents (Str. Pneumoniae, H.influenzae B, Neiss. meningitides, etc.)[13]. Although H.influenzae type B meningitis is most common, hearing loss occurs with higher frequency after pneumococcal meningitis in infants over one-month age.

A matter of dispute is the exact location of the damage in hearing loss caused by meningitis, whether it is in the hair cells, the terminal branches of the auditory nerve or the auditory nerve itself. Hair cells, terminal branches and the cell bodies of the nerve fibres are surrounded by perilymph and endolymph fluid. Medications and/or bacterial infections are the most probable causes for the damage of hair cells and the nerve [14]. Serial BAEP recordings during the first 48 hours from the beginning of their impact have demonstrated early occurrence of hearing loss. All changes in BAEPs disappear within two weeks from monitoring without registered cases of later hearing loss or recovery. This suggests that the functional status of peripheral neurons of auditory pathways is intact in children with congenital deafness compared with those who have lost their hearing after meningitis.

Hearing loss caused by bacterial meningitis starts during meningitis and the cases of hearing loss do not decrease after specific antibacterial treatment. Although, in some cases, acute otitis media with direct spread through the labyrinth window can precede the onset of the disease it is more likely bacterial toxins to penetrate through the cochlear aqueduct or through the internal auditory canal, thus leading to perineuritis or neuritis of CN VIII and/or purulent labyrinthitis. Other pathophysiological mechanisms participating in hearing loss include serous or toxic labyrinthitis, thrombophlebitis or embolisation of the labyrinth vessels, hypoxia or anoxia of CN VIII and the central auditory pathways.

In children with bilateral sensorineural hearing loss 
after bacterial meningitis, cochlear implantation is a successful remedy for rehabilitation of hearing. Surgical treatment has to be performed quickly because of labyrinthine ossification occurring as a complication of meningitis.

\section{Intake of ototoxic medications}

Hearing loss resulting due to ototoxic medications from the group of aminoglycosides such as gentamicin, and toxic substances such as lead, is a rare and preventable cause for sensorineural hearing loss. Some medications cause irreversible deafness, while intake of others leads to hearing loss which can be reversed when medications are stopped. There seems to be an idiosyncratic and genetic susceptibility to ototoxic medications [15]. Medication-induced hearing impairments are usually bilateral, symmetrical and of variable severity. Loss of hearing is progressive, initially affecting high frequencies.

Borradori et al [16] have identified ototoxicity closely related to a prolonged administration and higher total dose of ototoxic drugs, particularly aminoglycosides and furosemide. Salamy et al [17] find a high risk of hearing loss when furosemide is administered in the neonatal period in greater amounts, for longer durations and in combination with aminoglycosides.

Risk of hearing loss caused by intake of ototoxic medications also exists in patients with malignant tumours treated with cisplatin. Other medications known to induce sensorineural hearing loss are acetylsalicylic acid, chloramphenicol, chloroquine phosphate, dihydrostreptomycin, neomycin, nortriptyline, pharmacetin, polymyxin $\mathrm{B}$, streptomycin, vancomycin.

Hearing loss has been reported in association with prenatal exposure to alcohol, trimethadione, and methyl mercury as well as in prenatal iodine deficiency.

Neonatal wards differentiate a group of children who are at risk for hearing loss, combining the impact of factors such as ototoxic medications used during treatment in the units, extracorporeal membrane oxygenation and continuous ventilation and hyperbilirubinemia in some of the young patients.

\section{Stay in a neonatal intensive care unit}

It is clear from research that the most frequent risk factors for hearing loss in neonatal intensive care units are ototoxic medications (44,4\%), low weight at birth $(17,8 \%)$, assisted ventilation lasting 5 days (16,4\%), low Apgar scores (13,95\%) [18].
Hypoxia, hyperbilirubinemia and, to a lesser degree, exposure to ototoxic medications determine hearing loss in the neonatal intensive care unit.

Hypoxia varies from apnoea, low Apgar score or more often documented as $\mathrm{PO} 2<50 \mathrm{~mm} \mathrm{Hg}$.

Hyperbilirubinemia is a factor related with sensorineural hearing loss in neonatal intensive care units and also with auditory neuropathy. Specific deviations in the auditory evoked responses have been demonstrated in the presence of hyperbilirubinemia. These aberrations disappear after blood exchange transfusion and bilirubin levels return to normal. DeVries et al [19] have found that a higher mean duration of hyperbilirubinemia (48 hours versus 24 hours) and low birth weight is associated with higher risk of sensorineural hearing loss.

Persistent pulmonary hypertension and extracorporeal membrane oxygenation are indicators for hearing monitoring, performed every six months until the age of three, since delayed onset of sensorineural hearing loss has been reported.

Interruption of blood flow to the cochlea is another theorized cause for sudden hearing loss. It usually leads to cochlear fibrosis and ossification which are well-visualized on MRI.

\section{Acoustic trauma}

Loud noise is one of the main reasons for hearing loss. Although acoustic trauma can sometimes be considered a rough equivalent of noise-induced hearing impairment including chronic cases, their clinical course and treatment outcomes differ. Noise-induced hearing loss can be divided into acute and chronic and chronic is further divided into two types - occupational (in the majority of cases) and non-occupational.

Acute noise-induced hearing loss is also two categories: one is acoustic trauma and the other is acute noise-induced hearing loss (such as hearing loss related with listening to loud music at concerts). Acoustic trauma happens instantly, for example, with a gun-shot or an explosion. Immediately after the explosion, a mechanical injury is induced since the noise level physically exceeds the 'elastic limit' of the peripheral auditory mechanism. This type of trauma is caused by an extremely intense level of noise ( $\geqq 130 \mathrm{dBA})$. On the other hand, acute noise-induced hearing loss is usually caused after some exposure time, from several minutes to several hours. After attending a concert people might experience ringing sounds or muffled ears. This type of impairment is mainly caused by metabolic damage due to 'excitotoxicity' from exposure to intense sound of around 100-120 dBA. 
Outer hair cells in the cochlea when initially exposed to loud noise suffer from reversible damage which causes temporary threshold shift of hearing; if a person is exposed long enough, the damage remains permanent. Standard tone threshold audiometry does not record these early changes and until hearing loss can be detected by the audiogram, permanent damage is caused to the outer hair cells. Occupational guidelines for industry noise exposure require employees exposed to sound levels above $85 \mathrm{~dB}(\mathrm{~A})$ to be provided with personal protective equipment.

It has been proved that above $85 \mathrm{~dB}$ the likelihood for acquiring permanent damage of hair cells quickly increases, with a seemingly slight increase in the level of decibels. As logarithmic scales are used to measure decibels, for every 3-4 dB increase in sound pressure the permissible time for exposure to noise above $85 \mathrm{~dB}$ is halved [20].
Acute noise-induced hearing loss is considered to be reversible to some degree. Currently, several treatment methods are used in clinical practice for the recovery of hearing: steroids, hyperbaric oxygen therapy and dextran being the most widely spread.

Exposure to more than $140 \mathrm{~dB}$ peak sound pressure can cause an immediate, profound and permanent deafness [21].

\section{Head trauma}

It is well known that fractures of the temporal bones are associated with SNHL. Hearing loss resulting from concussion and traumatic brain injury is less often recognised in the absence of apparent temporal bone fractures.

Cochlear concussion presents with a PTA result from 2000 to $4000 \mathrm{~Hz}$ in the absence of temporal bone fracture or other organic pathology following the head trauma.

\section{References:}

1. Merchant S, Durand M, Adams J. Sudden deafness: is it viral? ORL J Otorhinolaryngol Relat Spec 2008;70:52-62.

2. Palacios, G. C., Montalvo, M. S., Fraire, M. I., Leon, E., Alvarez, M. T., \& Solorzano, F. (2008). Audiologic and vestibular findings in a sample of human immunodeficiency virus type-1-infected Mexican children under highly active antiretroviral therapy. International Journal of Pediatric Otorhinolaryngology,72(11), 1671-1681.

3. Prasad, H. K., Bhojwani, K. M., Shenoy, V., \& Prasad, S. C.(2006). HIV manifestations in otolaryngology. American Journal of Otolaryngology, 27(3), 179-185.

4. Rarey, K. E. (1990). Otologic pathophysiology in patients with human immunodeficiency virus. American Journal of Otolaryngology, 11(6), 366-369

5. Pappas, D. G. Jr., Chandra, H. K., Lim, J., \& Hillman, D. E.(1994). Ultrastructural findings in the cochlea of AIDS cases. American Journal of Otolaryngology, 15(4), 456-465.

6. Grimaldi, L. M., Luzi, L., Martino, G. V., Furlan, R., Nemni, R., Antonelli, A.,...Pozza, G. (1993). Bilateral eighth cranial nerve neuropathy in human immunodeficiency virus infection. Journal of Neurology, 240(6), 363-366

7. Hashimoto H, Fujioka M, Kinumaki H, Kinki Ambulatory Pediatrics Study Group. An office based prospective study of deafness in mumps. Pediatr Infect Dis J 2009;28(3):173-5

8. Fukuda, S., Ishikawa, K., \& Inuyama, Y. (1994). Acute measles infection in the hamster cochlea. Acta Otolaryngologica Supplement, 514, 111-116.

9. Dunmade, A. D., Segun-Busari, S., Olajide, T. G., \& Ologe, F. E. (2007). Profound bilateral sensorineural hearing loss in Nigerian children: Any shift in etiology? Journal of Deaf Studies and Deaf Education, 12(1), 112-118.

10. Sweeney, C. J., \& Gilden, D. H. (2001). Ramsay Hunt syndrome. The Journal of Neurology, Neurosurgery, and Psychiatry,71(2), 149-154.

11. Abramovich, S., \& Prasher, D. K. (1986). Electrocochleography and brain-stem potentials in Ramsay Hunt syndrome. Archives of Otolaryngology Head and Neck Surgery,112(9), 925-928

12. Murakami, S., Hato, N., Horiuchi, J., Honda, N., Gyo, K., \& Yanagihara, N. (1997). Treatment of Ramsay Hunt syndrome with acyclovirprednisone: Significance of early diagnosis and treatment. Annals of Neurology, 41(3), 353-357

13. Edmond K, Clark A, Korczak VS, et al. Global and regional risk of disabling sequelae from bacterial meningitis: a systematic review and meta-analysis. Lancet Infect Dis 2010;10(5):317-28

14. Wright T. 1999. Bacterial meningitis and deafness. 1986. Clin Otolaryngol 24:385-387.

15. Johnsonbaugh RE, Drexler HG, Light IJ, et al. 1974. Familial occurrence of drug-induced hearing loss.

16. Borradori C, Fawer CL, Buclin T, et al. 1997. Risk factors of sensorineural hearing loss in preterm infants. Biol Neonate 7:1-10

17. Salami A, Eldredge L, Tooley WH. 1989. Neonatal status and hearing loss in high- risk infants. J Pediatr 114:847-85.

18. Vohr BR, Widen JE, Cone-Wesson B, et al. 2000.Identification of neonatal hearing impairment: characteristics of infants in the neonatal intensive care unit and well-baby nursery. Ear Hear 21:373 -382.

19. DeVries LS, Lary S, Dubowitz LMS. 1985. Relationship of serum bilirubin levels to ototoxicity and deafness in high-risk low-birthweight infants. Pediatrics 76:351-354.

20. https://www.osha.gov/SLTC/noisehearingconservation/index.html\#standards

21. Brookhouser PE. Worthington DW, Kelly WJ 1992. Noise- induced hearing loss in children. Laryngoscope 102:645-655. 